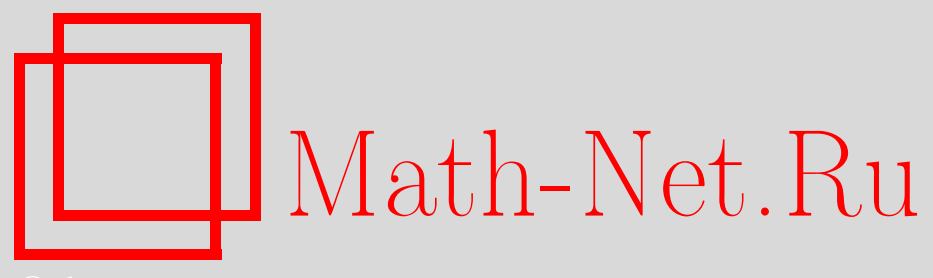

Ф. Носков, Бесконечность - не предел, Квант, 2019, номер 8, 44-47

DOI: https://doi.org/10.4213/kvant20190806

Использование Общероссийского математического портала Math-Net.Ru подразумевает, что вы прочитали и согласны с пользовательским соглашением http://www.mathnet.ru/rus/agreement

Параметры загрузки:

IP : 52.23 .180 .231

26 апреля 2023 г., 17:02:44

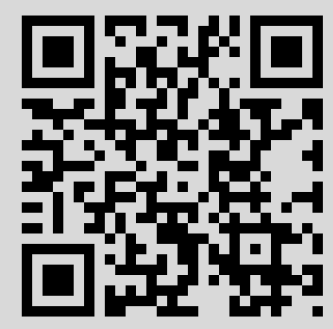




\section{Бесконечность - не предел}

\section{Ф. HOCKOB}

$\exists$ ТА СТАТЬЯ ПРЕДСТАВЛЯЕТ СОБОЙ ПО-

пытку дать первичное описание порядковых чисел (ординалов). Похожий предмет в «Кванте» уже обсуждался в статье А.Кириллова, И.Клумовой и А.Сосинского «Сюрреальные числа» (№11 за 1979 г.), однако тогда ординалы были упомянуты вскользь, отмечалась лишь их связь с бесконечными последовательностями. Примерно этой связью руководствовались и мы, и Кантор, когда их, собственно, открыл.

Среди комиксов известного в интернете автора под псевдонимом Дюран стоит отметить «Бесконечную шутку». Этот комикс великолепный; в частности, в нем главный герой рассказывает бесконечному числу математиков анекдот про, собственно, рассказчика анекдота. Это порождает некую цикличность, но суть не в этом. Суть в анекдоте

DOI: https://doi.org/10.4213/kvant20190806 про бесконечное число математиков, заходящих в бар. Вот он:

Заходит бесконечное число математиков в бар. Первый говорит бармену: «Мне кружку пива». Второй говорит: «Мне полкружки». Третий: «Мне четверть». И так далее. «Ну вас к черту», - восклицает бармен и наливает две кружки.

Пусть бармен обслужил бесконечное число математиков за 2 секунды. А именно, нулевой математик (а в дискретной математике принято отсчитывать от нуля) обслуживается за 1 секунду, первый - за полсекунды, второй - за четверть и так далее, $n$-й обслуживается за $\frac{1}{2^{n}}$ секунды. Итак, все математики будут обслужены за 2 секунды. После этого в бар заходит еще один математик.

Каждому из предыдущих математиков мы могли присвоить номер. Нет вопросов в том, какого математика считать вторым, какого нулевым, а какого - стотысячным. Но вот каким числом занумеровать этого нового математика, который зашел в бар? Присвоим ему номер $\omega$, и количество математиков перед ним также будем обозначать $\omega$. Да, это бесконечное число, но ничего страшного в этом нет. Следующего за ним математика назовем $(\omega+1)$-м, следующего за этим $(\omega+2)$-м и так далее. Пусть и эти математи-

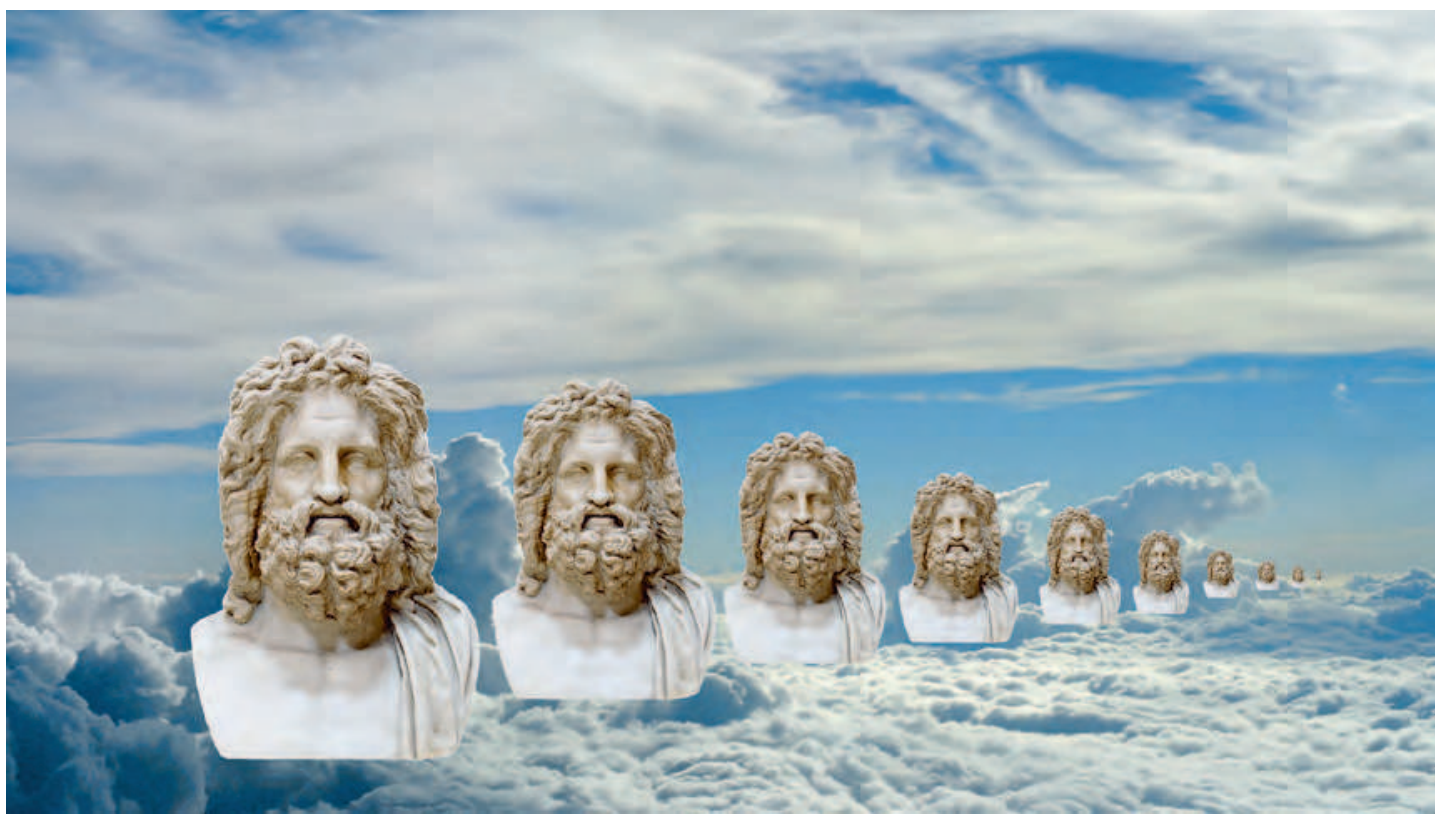


ки пройдут бар за 2 секунды так же, как это сделали первые $\omega$ математиков. Сколько же всего математиков прошло бар? Ответ прост: $\omega+\omega=\omega \cdot 2$. А пусть зайдут еще $\omega$ математиков! Теперь сколько их? $\omega \cdot 3$.

Хорошо, сделаем анекдот еще смешнее: теперь $\omega$ математиков заходят в бар за 1 секунду, потом еще $\omega$ математиков заходят в бар за полсекунды, потом еще $\omega$ математиков заходят в бар за четверть секунды и так далее. Сколько математиков окажется внутри бара за 2 секунды? Очевидно, $\omega \cdot \omega=\omega^{2}$.

Кажется, мы научились и складывать математиков, и умножать. Как же мы это делаем? Вот хотим сложить $\alpha$ математиков и $\beta$ математиков. Что это значит? Все просто: в бар зашли сначала $\alpha$ математиков, потом $\beta$ математиков, и их стало $\alpha+\beta$. Однако не все так просто. Рассмотрим следующую ситуацию: в бар сначала зашли два математика, а потом $\omega$ математиков. Но возможно ли отличить этот случай от того, когда в бар заходят $\omega$ математиков? Внимательно смотрим на рисунок 1 и понимаем, что нет (более точное обоснование требует введения понятия изоморфизма). Значит, $2+\omega=\omega$. А вот если в бар зайдет $\omega$ математиков, а за ними 2 математика, то ситуация будет совсем другой. Итак, $2+\omega$ не равно $\omega+2$. Тем самым, в общем случае $\alpha+\beta$ не равно $\beta+\alpha$, как мы привыкли.

Более интересным является введение умножения. Чтобы умножить 2 на 3, надо вместо каждого из трех математиков подставить двух и получить шесть математиков. Обобщим этот принцип на наш случай.

Скажем, мы хотим умножить $\omega$ на 2. Рассмотрим ситуацию: стояли два математи-

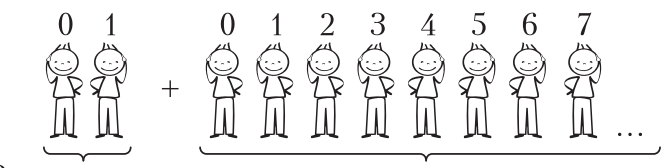

2 математика

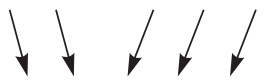

$\begin{array}{llllllllll}0 & 1 & 2 & 3 & 4 & 5 & 6 & 7 & 8 & 9\end{array}$

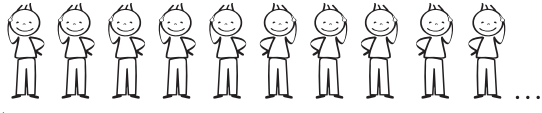

Pис. 1 ка. А мы вместо каждого поставили $\omega$ математиков, и вышло $\omega \cdot 2$ математиков (рис.2). В другом случае мы хотим умножить $\omega$ на

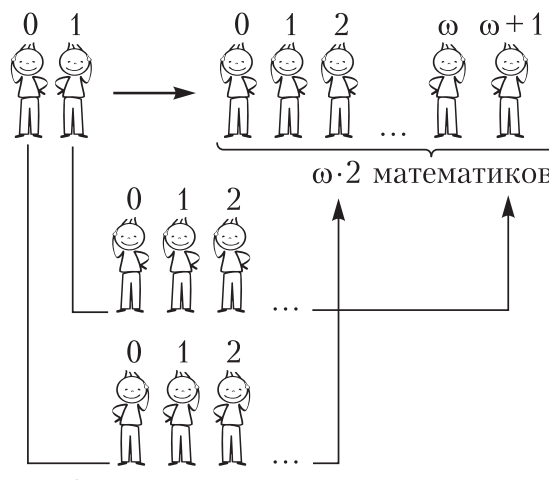

Pис. 2

$\omega$ : стояли $\omega$ математиков, мы вместо каждого математика поставили $\omega$ математиков. Таким образом, если мы хотим умножить $\alpha$ на $\beta$, мы просто вместо каждого математика из $\beta$ математиков подставляем $\alpha$ математиков.

Есть ли тут что-нибудь интересное? Оказывается, да: что будет, если мы 2 умножим на $\omega$ ? Подставим вместо каждого математика из $\omega$ математиков двух математиков (рис.3). Снова получим $\omega$ математиков. Получается, $\omega \cdot 2$ не равно $2 \cdot \omega$, т.е. $\alpha \cdot \beta$ не равно $\beta \cdot \alpha$ в общем случае.

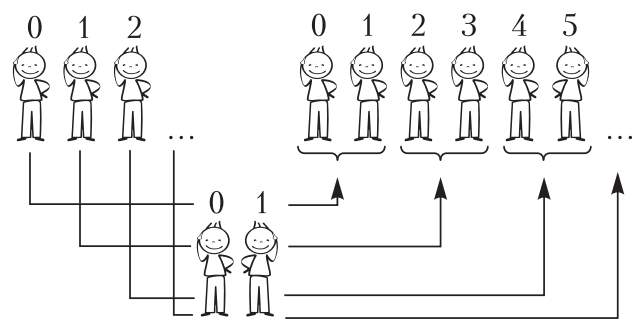

Pnc. 3

В качестве упражнения хочется предложить читателям доказать на математиках для произвольных $\alpha, \beta, \gamma$ такое соотношение: $\alpha \cdot(\beta+\gamma)=\alpha \cdot \beta+\alpha \cdot \gamma$, т.е. возможность раскрытия скобок слева. Еще интереснее обосновать некорректность раскрытия скобок справа, т.е. найти примеры, когда $(\beta+\gamma) \cdot \alpha \neq \beta \cdot \alpha+\gamma \cdot \alpha . \quad$ (Подсказка. Рассмотрите выражение $(\omega+1) \cdot 2$.)

Итак, было $\omega$ математиков, мы вместо каждого из $\omega$ математиков подставили $\omega$ математиков, получили $\omega^{2}$ математиков. 
После вместо каждого из $\omega^{2}$ математиков подставили $\omega$ математиков, получили $\omega^{3}$ математиков. Хорошо, я смогу получить хоть $\omega^{100}$. Как же определить $\omega^{\omega}$ математиков?

Представим сотворение нового, идеального мира. Жил на земле только один математик. Однажды на облаках в поднебесье собираются $\omega$ богов на совет. Говорит бог номер 0: «Да будет на земле вместо каждого математика $\omega$ математиков! $\omega$ математиков. Бог номер 1 говорит: «Да будет на земле вместо каждого математика $\omega$ математиков! И появилось на земле $\omega^{2}$ математиков. Бог номер 2 сказал: «Да будет на земле вместо каждого математи ка ш математиков!» И стало математиков на земле $\omega^{3}$. И каждый следуюший бог говорил: «Да будет на земле вместо каждого математика ш математиков! И стало на земле $\omega^{\omega}$ математиков. И поняли боги, ито это хорошо.

Что же сделали боги? Они $\omega$ раз умножили $\omega$ на само себя (чуть более детальное обоснование происходящего дано ниже мелким шрифтом). И получили $\omega^{\omega}$ математиков.

Теперь представим новую ситуацию. Жил на земле только один математик. В поднебесье собрался пантеон из $\omega^{\omega}$ богов. И сказал нулевой бог: «Да будет на земле вместо каждого математика а математиков!» А первый бог сказал: «Да будет на земле вместо каждого математика $\omega$ математиков!» И второй бог это сказал, и бог номер $\omega+1$ это сказал, и бог номер $\omega^{2}+\omega \cdot 2+4$ это сказал, так все боги, кроме нулевого, это сказали. И появилось на земле $\omega^{\omega^{\omega}}$ математиков. И поняли боги, ито это хорошо.

Итак, как нам получить $\alpha$ в степени $\beta$ математиков? Надо, чтобы на небесах сидели $\beta$ богов, на земле жил только один математик и каждый бог сказал: «Да будет на земле вместо каждого математика $\alpha$ математиков!» Что делают боги? Они $\beta$ раз умножают $\alpha$ на само себя. Делают то же, что обычно делаем в таких случаях мы, когда 2 возводим в куб.

Ключевым свойством ординалов (т.е. всех этих чисел, которыми мы считаем бесконечное число математиков) является возможность индукции по ним. Такая индукция называется трансфинитной. Рассмотрим подробнее одну из формулировок принципа математической индукции. Пусть $\varphi(n)$ - некоторое утверждение относительно натурального числа $n$ (к примеру, $\sum_{i=0}^{n} i=\frac{n(n+1)}{2}$ ), тогда принцип можно сформулировать так:

Если из того, что для любых т меньших, чем $n$, выполняется $\varphi(m)$, следует, что выполняется $\varphi(n)$, то для любого натурального п выполняется $\varphi(n)$.

Как таким принципом пользуются? Говорят: «Давайте предположим, что для всех натуральных $m<n$ выполняется $\sum_{i=0}^{m} i=\frac{m(m+1)}{2}$. Докажем тогда, что $\sum_{i=0}^{n} i=\frac{n(n+1)}{2}$ ». Заметим, что в такой формулировке база задается неявно: утверждение «для всех натуральных $m<0$ выполняется $\sum_{i=0}^{m} i=\frac{m(m+1)}{2} \gg$ является истинным просто потому, что таких $m$ нет. Таким образом, чтобы доказать утверждение для $n$, нам все равно надо рассмотреть два случая: 1) $n=0$; 2) существует натуральное число $n-1$, которое меньше $n$, а значит, для него верно $\sum_{i=0}^{n-1} i=\frac{(n-1)((n-1)+1)}{2}$. После того, как мы доказали утверждение для $n$ в двух этих случаях, мы получаем посылку принципа индукции: «Из того, что для любых $m$ меньших, чем $n$, выполняется $\varphi(m)$, следует, что выполняется $\varphi(n)$ ». Зная, что принцип индукции истинен, мы заключаем, что для любого натурального $n$ верно $\sum_{i=0}^{n} i=\frac{n(n+1)}{2}$.

Однако точно таким же образом мы можем идти индукцией по математикам. Пусть стоят $\alpha$ математиков и про математика номер $\beta$ мы можем сказать какое-то утверждение $\varphi(\beta)$. Пусть нам известно, что из того, что для любого математика с номером $\gamma$ меньшим, чем $\beta$, справедливо $\varphi(\gamma)$, следует, что справедливо $\varphi(\beta)$. Тогда по принципу индукции утверждение $\varphi$ справедливо для всех $\alpha$ математиков.

Иногда мы говорим о рекурсии или об индуктивном определении. Примером может послужить определение возведения в натуральную степень:

1. $a^{0}=1$,

2. $a^{n+1}=a^{n} \cdot a$.

В действительности не очевидно, отчего мы сможем посчитать $a^{n}$ для любого натурального $n$ и произвольного $a$ в результате такого определения. Возможность рекурсии следует из справед- 
ливости самого принципа индукции. Таким образом, в нашем примере с богами мы «дали» индуктивное определение возведения в степень, заметя под ковер один важный момент. Рассмотрим три случая:

1. В пантеоне нет богов, а на земле 1 математик. Выходит, что $a^{0}=1$.

2. В пантеоне $\beta+1$ богов. Тогда после слов последнего бога появится $\alpha^{\beta+1}=\alpha^{\beta} \cdot \alpha$.

3. В пантеоне $\alpha$ богов, причем нет такого ординала $\gamma$, что $\alpha=\gamma+1$. Примером такого $\alpha$ является $\omega-$ у этого «числа» нет предыдущего. В таком случае говорят о взятии супремума (sup) по всем меньшим ординалам. Говоря о супремуме, мы имеем в виду некоторое слияние последовательностей математиков. Пример получения $\omega$ математиков таким способом показан на рисунке 4.

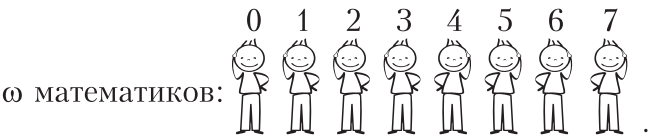

0 математиков:

\section{$\uparrow$}

1 математик:
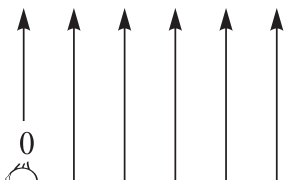

3 математика:

2 математика:

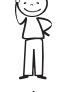

0
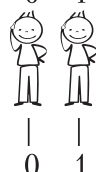

$$
\begin{array}{lll}
0 & 1 & 2
\end{array}
$$

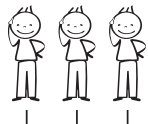

0

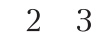

4 математика:
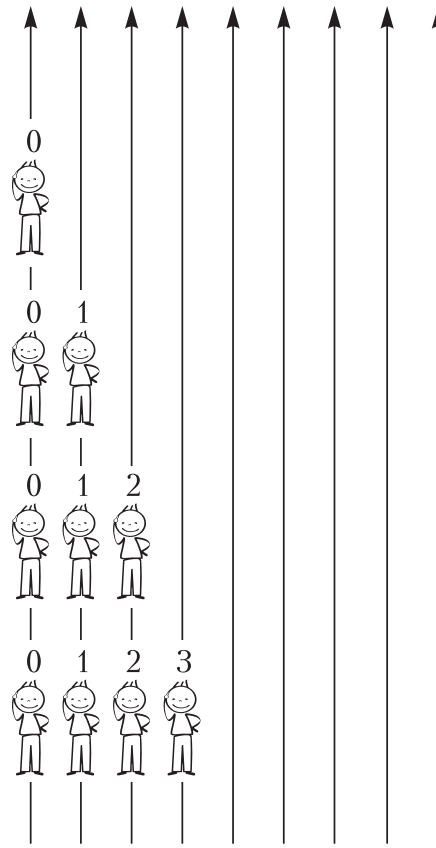

Рис. 4

В результате такой операции должна получиться наименьшая последовательность, содержащая все конечные последовательности математиков в качестве своих начальных подпоследовательностей. Таким образом мы хотим сказать, что $\omega=\sup \{\gamma \mid \gamma-$ конечный ординал $\}$.

Похожим образом определяется степень в случае ординала, не имеющего предыдущего, т.е. $\delta^{\alpha}=\sup \left\{\delta^{\gamma} \mid \gamma<\alpha\right\}$. Напомним, что в результате получается слияние последовательностей, созданных предыдущими богами, описанным на рисунке 4 способом.

Итак, мы определили возведение в большую степень через возведение в меньшие. Сославшись на возможность трансфинитной рекурсии, поймем, что таким образом мы научились возводить ординалы в любую степень.

Сейчас мы подходим к финалу истории. Вот ее окончание:

В поднебесъе сидели ш богов. И нулевой бог сказал: «Да будет на земле ш математиков!» И первый бог сказал: «Да будет на земле $\omega$ математиков в такой степени, в какой их уже на земле есть! » И второй бог это сказал, и третий бог это сказал, и все следуюшие боги это сказали. И стало на земле $\varepsilon_{0}$ математиков. И поняли боги, что это хорошо.

Но позавидовал дьявол тому, что делали боги. Пришел в поднебесье и сказал: «Да будет на земле $а$ математиков в такой степени, в какой их уже есть на земле! » Но математиков осталось все равно $\varepsilon_{0}$. Так дьявол был посрамлен.

Что же произошло в этой истории? После слов нулевого бога на земле $\omega$ математиков, после слов первого $\omega^{\omega}$ математиков, после слов второго $\omega^{\omega^{\omega}}$, после слов третьего $\omega^{\omega^{\omega^{\omega}}}$ и так далее. «Число», которое получилось в результате, называется $\varepsilon_{0}$. Оно обладает одним замечательным свойством: $\omega^{\varepsilon_{0}}=\varepsilon_{0}$.

Bсе эти бесконечные числа называются ординалами. В действительности они обозначают специальный вид порядка на различных множествах, о чем мы здесь распространяться не будем. Подробнее об этом можно прочитать в книге Н.К.Верещагина и А.Шеня «Начала теории множеств» (М.: МЦНМО, 1999). 